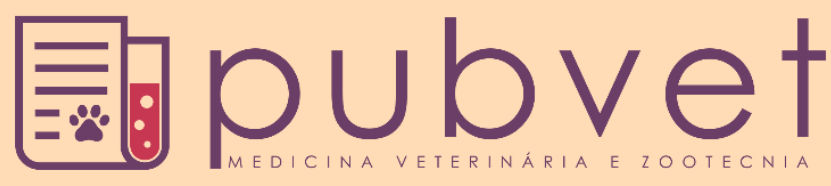

https://doi.org/10.31533/pubvet.v15n02a758.1-10

\title{
Aspectos epidemiológicos, clínicos, patológicos, diagnóstico, profilaxia e tratamento da hipocalcemia em bovinos: Revisão
}

\author{
Luan Henrique Fabris ${ }^{1 *}$, Júlia Marchioro ${ }^{1}$, Keli Daiane Cristina Libardi Ramella ${ }^{2}$ \\ ${ }^{I}$ Discentes do curso de Medicina Veterinária da Pontifícia Universidade Católica do Paraná - Toledo, Brasil. \\ ${ }^{2}$ Docente Pontifícia Universidade Católica do Paraná Toledo, Brasil \\ *Autor para correspondência, E-mail: luanhenriquefabris@hotmail.com
}

\begin{abstract}
Resumo. A hipocalcemia é uma doença metabólica relativamente comum em vacas leiteiras durante o período de transição, momento em que ocorrem profundas modificações hormonais, anatômicas e fisiológicas no organismo animal visando preparar a fêmea para o parto e posterior produção de leite. A referida pode ocorrer de duas formas: a forma subclínica, quando os valores de cálcio sérico se situam abaixo de $8,5 \mathrm{mg} / \mathrm{dL}$ e a forma clínica quando dos valores de cálcio abaixo de $5,5 \mathrm{mg} / \mathrm{dL}$. Os prejuízos causados à bovinocultura de leite são consideráveis, sobretudo na forma subclínica de ocorrência, em que a ausência de demonstração de sintomatologia clínica dificulta o diagnóstico. A doença geralmente ocorre em torno de 24 a 48 horas após o parto, onde se há um decréscimo nos níveis séricos do cálcio circulantes, uma vez que se tem uma grande demanda para produção do colostro e para o parto. O diagnóstico clínico baseia-se no histórico e nos sinais clínicos do animal, e através da mensuração do mineral no sangue. A terapêutica clínica com soluções à base de cálcio demonstra bons resultados a campo quando do diagnóstico rápido e preciso. Entretanto, vale ressaltar que a profilaxia é a melhor opção, melhorada por um bom manejo nutricional no período seco e de transição, pelo acompanhamento do ECC, emprego de dietas aniônicas concomitantemente ao monitoramento da acidificação do $\mathrm{pH}$ urinário. Ademais, tem sido citada como uma alternativa para prevenção da hipocalcemia, o fornecimento de drench para vacas periparturientes como uma das alternativas de profilaxia à doença. A presente revisão objetiva elencar a etiologia, sintomatologia clínica, diagnóstico, tratamento e profilaxia da hipocalcemia em bovinos leiteiros.
\end{abstract}

Palavras chave: bovinos leiteiros, hipocalcemia, período de transição.

\section{Epidemiological, clinical, pathological aspects, diagnosis, prophylaxis and treatment of hypocalcemia in cattle: Review}

Abstract. Hypocalcemia is a relatively common metabolic disease in dairy cows during
the transition period, a time when profound hormonal, anatomical and physiological
changes occur in the animal body in order to prepare the female for delivery and later milk
production. This can occur in two ways: the subclinical form, when the serum calcium
values are below $8.5 \mathrm{mg}$ / dL and the clinical form when the calcium values are below 5.5
$\mathrm{mg} / \mathrm{dL}$. The damage caused to dairy cattle is considerable, especially in the subclinical
form of occurrence, in which the absence of demonstration of clinical symptoms makes
diagnosis difficult. The disease usually occurs around 24 to 48 hours after delivery, where
there is a decrease in circulating serum calcium levels, since there is a great demand for
colostrum production and delivery. The clinical diagnosis is based on the animal's history
and clinical signs, and by measuring the mineral in the blood. Clinical therapy with
calcium-based solutions shows good results in the field when the diagnosis is quick and 
accurate. However, it is noteworthy that prophylaxis is the best option, improved by good nutritional management in the dry and transitional period, by monitoring the ECC, using anionic diets concurrently with the monitoring of urinary $\mathrm{pH}$ acidification. In addition, it has been cited as an alternative for the prevention of hypocalcemia, the provision of drench for periparturient cows as one of the prophylaxis alternatives to the disease. This review aims to list the etiology, clinical symptoms, diagnosis, treatment and prophylaxis of hypocalcemia in dairy cattle.

Keywords: Dairy cattle, hypocalcemia, transition period

\title{
Aspectos epidemiológicos, clínicos, patológicos, diagnóstico, profilaxis y tratamiento de la hipocalcemia en vacas: Revisión
}

\begin{abstract}
Resumen. La hipocalcemia es una enfermedad metabólica relativamente común en las vacas lecheras durante el período de transición, un momento en el que ocurren profundos cambios hormonales, anatómicos y fisiológicos en el cuerpo del animal con el fin de preparar a la hembra para el parto y la posterior producción de leche. Esto puede ocurrir de dos formas: la forma subclínica, cuando los valores de calcio sérico están por debajo de 8,5 $\mathrm{mg}$ / dL y la forma clínica cuando los valores de calcio están por debajo de 5,5 mg / dL. El daño causado al ganado lechero es considerable, especialmente en la forma subclínica de aparición, en la que la ausencia de demostración de síntomas clínicos dificulta el diagnóstico. La enfermedad generalmente ocurre alrededor de las 24 a 48 horas después del parto, donde hay una disminución en los niveles de calcio sérico circulante, ya que existe una gran demanda de producción y entrega de calostro. El diagnóstico clínico se basa en la historia y los signos clínicos del animal y en la medición del mineral en la sangre. La terapia clínica con soluciones a base de calcio muestra buenos resultados en el campo cuando el diagnóstico es rápido y preciso. Sin embargo, es de destacar que la profilaxis es la mejor opción, mejorada por un buen manejo nutricional en el período seco y de transición, mediante el monitoreo de la CEC, utilizando dietas aniónicas de manera concurrente con el monitoreo de la acidificación del pH urinario. Además, se ha citado como alternativa para la prevención de la hipocalcemia, la provisión de empapamiento a las vacas periparturientas como una de las alternativas de profilaxis a la enfermedad. Esta revisión tiene como objetivo enumerar la etiología, los síntomas clínicos, el diagnóstico, el tratamiento y la profilaxis de la hipocalcemia en el ganado lechero.
\end{abstract}

Palabras clave: Ganado lechero, hipocalcemia, período de transición

\section{Hipocalcemia em vacas leiteiras}

O potencial agropecuário brasileiro é inegável e reconhecido mundialmente, englobando-se neste contexto, a bovinocultura de leite. De acordo com dados do IBGE (2019), a produção leiteira no Brasil em 2018 alcançou o montante de 24,46 bilhões de litros. Entre 1997 e 2018, a produção nacional teve um incremento de 13,77 bilhões de litros, tal alavanco produtivo representa um aumento de $56,29 \%$ na produção leiteira no país em 21 anos.

Vilela et al. (2002) destacaram que a melhoria na produtividade das vacas leiteiras obteve um papel mais impactante no aumento da produção do que o aumento no número de animais no rebanho nacional, ou seja, houve um grande aumento na produtividade por vaca. Conquanto, vale salientar que essa maior produção por vaca obtida por intermédio de um melhoramento genético e nutricional possui correlação positiva com uma maior predisposição dos animais à problemas metabólicos e sanitários (Ingvartsen \& Moyes, 2015).

É dito que a maior concentração de doenças em vacas leiteiras ocorre no período pré e pós-parto, também conhecido como período de transição. Nesse período, as alterações hormonais, alterações na fisiologia e na anatomia corporal da fêmea bovina para posterior parto e produção de leite, predispõem os animais a distúrbios anômalos, resultando em aumento na ocorrência de doenças, aumento na taxa de 
descarte, decréscimo produtivo e baixo desempenho reprodutivo (Hayirli et al., 2002; Huzzey et al., 2007).

A hipocalcemia consiste em um distúrbio comum em vacas leiteiras e impacta significativamente na produção leiteira, sendo que, em casos graves, pode culminar na morte do animal. De acordo com Martín-Tereso \& Martens (2014), a concentração sérica normal de cálcio em uma vaca adulta situa-se acima de 2,0 mmol/L de sangue, correspondendo a 8,5 a $10,5 \mathrm{mg} / \mathrm{dL}$ e de cálcio ionizável de 4,5 a 5,5 $\mathrm{mg} / \mathrm{dl}$ (Jawor et. al., 2012; Oetzel, 2013). É dito que, devido à necessidade de produção de colostro, fenômeno fisiológico na fêmea leiteira, ocorre uma diminuição nas concentrações de cálcio sérico em torno de 12 a 24 horas após o parto, uma vez que tal componente possui praticamente o dobro do elemento quando comparado ao leite normal. Além disso, há um alto requerimento do mineral para formação do feto e produção de leite, o que culmina em uma alta demanda e diminuição dos níveis séricos do elemento (Goff, 2008; Goff, 2014).

Sabe-se que vacas acometidas por hipocalcemia, ficam predispostas a uma gama de enfermidades metabólicas e infecciosas, sobretudo no período de transição, dentre tais doenças, destacam-se as metrites, mastites, retenção de placenta, deslocamento de abomaso (Curtis et al., 1983; Goff, 2008; Martinez et al., 2012). Dados epidemiológicos e observações de campo mostraram que a incidência da hipocalcemia clínica oscila em torno de 3 a 15\%, sendo que a forma subclínica pode vir a acometer até $50 \%$ de um rebanho leiteiro (Reinhardt et al., 2011). Estudos recentes denotaram que vacas com hipocalcemia apresentam a função imunológica suprimida, o que justifica o aumento da susceptibilidade à demais enfermidades (Martinez et al., 2014).

A literatura vigente ressalta que vacas com hipocalcemia, apresentam níveis de ácidos graxos não esterificados (NEFA) (acima de 0,6 mmol/L) e de Beta-hidroxibutirato (BHB) elevados, (acima de 15 $\mathrm{mg} / \mathrm{dL}$ ) indicativo de que há um aumento da lipomobilização em casos da deficiência do elemento (Goff, 2008; Van Cleef, 2009; Martinez et al., 2012; Santos, 2011) Martinez et al. (2014) atestaram a informação supracitada mediante indução iatrogênica da doença.

Estudos apontam que a indução da hipocalcemia em vacas leiteiras resulta na diminuição do consumo de matéria seca (CMS) e dos níveis séricos de insulina, além de diminuição no consumo de alimentos concentrados e da ruminação favorecendo a acidose ruminal. Ademais, vacas com hipocalcemia apresentam grandes probabilidades do desenvolvimento de distúrbios como deslocamento de abomaso, retenção de placenta e prolapsos uterinos particularmente em vacas leiteiras multíparas e de alta produtividade (Chapinal et al., 2011; Curtis et al., 1983; Goff, 2008; Wu et al., 2008). O que se acredita é que tais anormalidades ocorram devido aos baixos níveis de Cálcio ionizável $\left(\mathrm{Ca}^{2+}\right)$, que é prontamente disponível, diminuindo as contrações da musculatura lisa.

De acordo com González \& Silva (2017), a hipocalcemia possui uma predisposição racial, afetando principalmente bovinos da raça Jersey, uma vez que estes animais possuem uma alta concentração de cálcio tanto no leite quanto no colostro. Além disso, é dito que possuem um menor número de receptores para este elemento. Nesse mesmo contexto, é dito que vacas da raça holandesa de alta produtividade também são grandemente acometidas, sendo que o risco se agrava com o aumento do número de lactações.

Esta revisão tem por intuito descrever sobre as causas da hipocalcemia em vacas leiteiras, o impacto econômico produzido, sinais clínicos, prevenção, controle e tratamento desta enfermidade tão impactante na bovinocultura leiteira nacional e mundial.

\section{Metabolismo do cálcio}

Em termos quantitativos, sabe-se que o cálcio compõe de 1 a $2 \%$ do organismo animal, responsável por funções relacionadas à mineralização dos ossos, coagulação sanguínea, regulação do metabolismo, transmissão de impulsos nervosos e também pelas contrações musculares (González et al., 2000).

O cálcio está presente na circulação sanguínea sob duas formas, sendo na forma ionizada e na forma orgânica. Cerca de $45 \%$ da concentração total de cálcio sérico está na forma ionizada $\left(\mathrm{Ca}^{2+}\right)$, sendo a fração biologicamente ativa ou utilizável pelo organismo. Considera-se que o organismo animal se 
encontra em homeostase quando as concentrações do elemento estejam dentro do limiar biologicamente estabelecido de 4,4 a 5,6 mg/dL (González et al., 2000; Littledike \& Goff, 1987).

A homesostase do cálcio no organismo é regida por dois hormônios, o paratormônio (PTH) secretado pelas glândulas paratireoides e a Calcitonina, secretada pelas células parafoliculares da glândula tireóide, respectivamente. Quando há depleção dos níveis séricos de cálcio ionizável $(\mathrm{Ca} 2+)$, a paratireoide secreta o paratormônio. Este, possui receptores em osteoblastos, enterócitos e células epiteliais renais. A síntese do hormônio, resulta no aumento da reabsorção óssea do mineral, aumento da absorção intestinal e da reabsorção renal do Cálcio contido no filtrado glomerular. Em contrapartida, à medida que os níveis séricos de cálcio ionizável aumentam, as células parafoliculares da tireoide secretam a calcitonina, resultando no aumento da deposição óssea, excreção renal e redução na reabsorção intestinal do elemento (Berchielli et al., 2011).

De acordo com a literatura as concentrações séricas de magnésio possuem uma correlação com a ocorrência de hipocalcemia em vacas leiteiras, uma vez que pode ocorrer hipomagnesemia concomitante à hipocalcemia no período transicional. Ademais, é dito que o magnésio auxilia na manutenção da homeostase do cálcio durante o período transicional, sendo citado que a ingestão desse microelemento possa auxiliar na prevenção da ocorrência clínica da doença (Moreira et al., 2015; Oetzel, 2012). Esse microelemento representa cerca de $0,045 \%$ do organismo animal e atua cofator de mais de 300 enzimas, na atividade neuromuscular e na composição da matriz óssea (González, 2000).

\section{Hipocalcemia dos bovinos leiteiros}

Tratando-se especificamente da hipocalcemia, é dito que a doença se manifesta, em sua grande maioria, nas primeiras 48 horas após o parto, mas pode se manifestar imediatamente antes do referido ou em até 72 horas após. De acordo com Riet-Correa et al. (2007), durante a gestação, a exigência do macro mineral é pequena pela fêmea, não obstante, à medida que se aproxima do parto, essa exigência aumenta, uma vez que se tem o início da produção de leite. Vale ressaltar que os mecanismos fisiológicos de absorção intestinal e reabsorção óssea necessitam de um período de 24 a 48 horas para se estabelecerem de forma efetiva, culminando na ocorrência da enfermidade.

A literatura ressalta que durante o periparto, todas as vacas leiteiras são acometidas pela hipocalcemia, em que os níveis séricos de cálcio estão abaixo de $8,5 \mathrm{mg} / \mathrm{dL}$. Entretanto, em torno de 5 a $20 \%$ dos casos, ocorrem de forma grave com demonstração evidente de sinais clínicos (Schafhäuser Jr, 2006; Smith, 2006).

Há um consenso de que, na forma subclínica (concentrações de cálcio sérico abaixo de 8,5 mg/dL) (Oetzel, 2013), devido à ausência de sintomatologia clínica evidente, o impacto econômico causado é maior, uma vez que o diagnóstico se torna mais laborioso. A ocorrência da enfermidade na forma subclínica resulta em menor consumo de matéria seca (CMS) durante início da fase lactacional e torna a fêmea mais propensa ao desenvolvimento de comorbidades como o deslocamento de abomaso, atonia ruminal, retenção de placenta, síndrome da vaca caída, metrites, baixo desempenho reprodutivo e mastites (Berchielli et al., 2011; Silveira et al., 2009).

Oliveira et al. (2011) discorreu que as vacas leiteiras demonstram sintomatologia clínica quando os níveis totais de cálcio séricos se encontram abaixo de $5,5 \mathrm{mg} / \mathrm{dl}$. A hipocalcemia clínica é dividida em três fases. Na primeira fase, as concentrações séricas do elemento encontram-se em torno de 5,5 a 7,5 $\mathrm{mg} / \mathrm{dl}$, sendo que o animal permanece em estação e apresenta sinais como dispneia, tremores musculares, excitação, ataxia e mugidos. Animais no segundo estágio apresentam concentrações de cálcio entre 3,5 a 6,5 mg/dl. O animal não suporta manter-se em estação, conquanto ainda é capaz de manter-se em decúbito esternal. Observa-se alterações em parâmetros vitais como taquicardia e temperatura corpórea reduzida $\left(36\right.$ a $\left.38^{\circ} \mathrm{C}\right)$, extremidades frias, depressão, anorexia e muflo seco. Smith, (2006) cita que o decúbito esternal com a cabeça voltada ao flanco caracteriza animais hipocalcêmicos. O terceiro estágio da doença é caracterizado por sintomatologia mais grave, com flacidez muscular completa, irresponsividade a estímulos, decúbito lateral e coma. Os níveis de cálcio sérico podem estar ao redor de $2 \mathrm{mg} / \mathrm{dl}$. O prognóstico desse estágio é extremamente ruim, podendo os animais ter poucas horas de vida ( Radostits et al., 2002; Smith, 2006). 
Pires et al. (2011) relatou que a concentração do cálcio no colostro é extremamente maior que no leite normal (quase duas vezes mais). $\mathrm{O}$ autor discorreu que, no pós parto-recente, uma fêmea bovina produzindo em torno de 10 litros de colostro por ordenha secretará em torno de 23 gramas de cálcio. Esse valor é de 8 a 10 vezes superior à quantidade de cálcio sérico em uma vaca de $600 \mathrm{~kg} / \mathrm{PV}$. Assim, para manter a homeostase, a exigência de cálcio de uma vaca leiteira produzindo 10 litros de leite em sua primeira ordenha, aumentaria suas exigências de cálcio ionizável de 20 gramas/dl no período seco para 43 gramas/dl no período lactacional. Essa mudança abrupta culminaria e justificaria a ocorrência de hipocalcemia subclínica na grande maioria das fêmeas bovinas (Santos, 2011).

\section{Diagnóstico e tratamento}

O diagnóstico se dá por meio do histórico e dos sinais clínicos apresentados, bem como a nível laboratorial, pela mensuração dos níveis do mineral no sangue. Nesse contexto, salienta-se que nível sérico do cálcio total em casos de hipocalcemia geralmente estão abaixo de $1,2 \mathrm{mmol} / \mathrm{L}(5 \mathrm{mg} / \mathrm{dL})$ podendo chegar, em casos extremos, a $0,5 \mathrm{mmol} / \mathrm{L}$ ( $2 \mathrm{mg} / \mathrm{dL}$ ) (Radostits et al., 2002).

A terapêutica clínica padrão consiste na administração do Borogluconato de cálcio (BGC) em solução de 20 a $30 \%$ tendo a via endovenosa como preferencial, na dose de na dose de um grama de Ca para cada $45 \mathrm{~kg}$ de peso vivo (Radostits et al., 2002). De acordo com Ortolani (1995), o êxito na terapêutica clínica tem relação direta com a dose de cálcio administrada, sendo que quanto mais alta a dose, maiores as chances de recuperação.

De acordo com Goff (1999), a administração de cálcio injetável por via subcutânea apresenta algumas limitações na resposta ao tratamento. A absorção de cálcio subcutâneo requer uma perfusão periférica adequada, o que pode reduzir a eficácia do tratamento em animais desidratados. Ademais, não é recomendado administrar mais de $75 \mathrm{~mL}$ de solução por ponto de aplicação, uma vez que o cálcio injetável provoca irritação, podendo levar a necrose tecidual.

O cálcio pode também ser administrado também por via peritoneal, sendo tal via a preferível em casos de toxemia (Radostits et al., 2002).

A dose de cálcio administrada em vacas hipocalcêmicas é de cerca de 8 a $12 \mathrm{~g}$, o que é baixa quando comparada à exigência diária do elemento. Entretanto, a terapia contribui para retorno do equilíbrio do cálcio no organismo e para o aumento das concentrações sanguíneas do mineral (Liesegang, 1998). É dito que o retorno à homeostase do cálcio ocorre em torno de 2 a 3 dias (Ogilvie, 2000).

A dose máxima do Borogluconato de cálcio - BGC por via endovenosa é de $250 \mathrm{ml}$ quando da solução a $40 \%$, sendo que devido ao risco de toxicidade do cálcio, muitos veterinários administram uma dose menor, reduzindo a eficácia do tratamento (Radostits et al., 2002).

Sabe-se que $\mathrm{Ca}^{++}$é cardiotóxico, sendo que a administração deve ser realizada em um período de 1020 minutos, sendo acompanhada de ausculta cardíaca. Na maioria das vacas a recuperação acontece imediatamente após o tratamento ou em até 2 horas. É dito que algumas vacas voltam a apresentar sinais 24-48 horas após o tratamento inicial e devem ser tratados uma segunda vez (Riet-Correa et al., 2001).

De acordo dados observados a campo, cerca de $85 \%$ dos animais respondem de forma satisfatória ao tratamento, sendo os sinais de melhora rapidamente observados (eructação, tremores musculares, defecação, sudorese no focinho), sendo que grande parte dos animais se levantam em até 10 minutos após a administração do BGC. Conquanto, há animais que podem demorar até 4 horas para se levantar. Caso o animal não se levante dentro de 5 ou 6 horas, deve-se realizar um novo exame e, caso necessário, administrar mais 8 a $12 \mathrm{~g}$ do elemento por via endovenosa (Radostits et al., 2002).

Substâncias como o magnésio e o fósforo inorgânico podem ser administradas juntamente com o cálcio em casos de hipocalcemia. Soluções à base de cálcio e magnésio podem ser administradas em casos de hipomagnesemia visando prevenir taquicardias e arritmias. Já o fosforo inorgânico pode ser empregado em casos de animais que não se levantam ou recaídas (Kronfeld, 1980; Caple, 1991).

Casos de superdosagem por cálcio englobam um tratamento prévio por parte do produtor, associado terapêutica empregada pelo veterinário (Eddy, 1999; Radostits et al., 2002). Concentrações elevadas de cálcio deprimem o sistema nervoso, diminuindo reflexos. O excesso de cálcio reduz contrações das 
paredes musculares e redução de apetite em se tratando do sistema gastrointestinal. No sistema cardíaco, diminui intervalo entre sístole e diástole e promove depressão cardíaca e respiratória (Dukes, 1996).

Ainda dentro do contexto da terapêutica clínica, sabe-se que a administração oral de cálcio consiste em uma opção sem risco de efeitos colaterais cardiotóxicos e pode ser utilizada em casos leves de paresia puerperal. Smith, 2006 salienta que a administração oral de 50g de Ca solúvel fornece cerca de 4g de $\mathrm{Ca}$ no sangue. $\mathrm{O}$ mesmo autor discorreu ainda que quando casos não complicados da doença são diagnosticados precocemente e a terapêutica é empregada de forma rápida, as chances de recuperação e retorno à produção de leite são excelentes.

\section{Profilaxia e controle}

De acordo com Oetzel (2013), Goff et al. (2014) e Farnia et al. (2018), a profilaxia da enfermidade engloba um correto manejo nutricional durante o período seco e de transição pré-parto, como a manipulação mineral na dieta, emprego de dietas aniônicas, monitoramento de $\mathrm{Ca}, \mathrm{Mg}$ e $\mathrm{K}$ nas dietas pré-parto, suplementação de fontes de vitamina $\mathrm{D}$ e a suplementação de cálcio por via oral, podendo tais medidas ser empregadas de forma associada ou isolada. Além disso, a realização de ordenha no préparto, controle do escore de condição corporal das vacas e controle da ingestão de carboidratos em vacas periparturientes também são medidas adicionais louváveis na prevenção da enfermidade.

A utilização de dietas aniônicas fundamenta-se na diferença catioaniônica da dieta (DCAD). Nesse caso, ocorre incremento nas concentrações de cloro e enxofre (ânions) e redução do sódio e potássio (cátions) na alimentação. Assim, ocorre o desencadeamento de uma leve acidose metabólica, alcançado por conta da ingestão de minerais aniônicos e redução do potássio na dieta. Para manter a homeostase, o bicarbonato $\left(\mathrm{HCO}_{3}\right)$ é secretado pelo tecido ósseo, objetivando neutralizar o $\mathrm{pH}$ sanguíneo, sendo o cálcio secretado de forma concomitante e aumentando seus níveis séricos. Porém, o maior desafio dessa dieta vem a ser fatores relacionados à palatabilidade do alimento (Albani \& Silva, 2017; Corbellini, 1998).

No Brasil, devido à utilização em larga escala de fósforo e potássio na adubação de forrageiras, tornase difícil a formulação de dietas visando a remoção de cátions da alimentação (Bernardi et al., 2002). Nesse contexto, Goff et al., (2004) e Wilkens et al., (2012) afirmam que uma forma alternativa de se alcançar uma diferença cátion aniônica negativa nas dietas consiste na inclusão de sais aniônicos como o cloreto de amônio $(\mathrm{NH} 4 \mathrm{Cl})$, cloreto de cálcio $(\mathrm{CaCl} 2)$, cloreto de magnésio $(\mathrm{MgCl} 2)$, sulfato de magnésio (MgSO4), sulfato de amônio (NH4)2SO4) e sulfato de cálcio (CaSO4) sob a forma de ração total misturada (RTM).

Ao promover alteração no $\mathrm{pH}$ sanguíneo, ocorre, por conseguinte, diminuição do pH urinário. Sendo assim, o monitoramento do $\mathrm{pH}$ urinário pré-parto é um método eficiente para determinação da resposta das vacas leiteiras em decorrência da dieta aniônica incrementada nos 21 dias que antecedem o parto (Lean et al., 2006). É dito que valores ideais de pH urinário na semana que antecedem o parto devem estar entre 6,2 e 6,8, sendo que valores inferiores aos elencados podem promover casos graves de acidose metabólica e acima, indicar falhas nas formulações, culminando em baixa eficácia das dietas (Mulligan et al., 2008).

Diversos trabalhos demonstraram que esta metodologia é eficiente na avaliação da funcionalidade da acidificação da dieta aniônica e, por conseguinte, na prevenção de doenças metabólicas em vacas periparturientes (Leite et al., 2003; Overton \& Waldron, 2004). Frigotto et al. (2010), ao monitorar o pH urinário pré-parto de vacas leiteiras que recebiam dietas formuladas para serem aniônicas, observaram que o $\mathrm{pH}$ médio foi de 8,4, contanto, o nível sérico médio de cálcio nos primeiros 10 dias de lactação esteve acima do limite mínimo para a ocorrência de hipocalcemia subclínica, confirmando efetividade da dieta aniônica.

Ademais, ressalta-se que outra importante ferramenta para o controle do manejo nutricional de rebanhos leiteiros e monitoramento de vacas no pós-parto recente consiste na avaliação do escore de condição corporal (ECC). Preconiza-se que vacas devem parir com um ECC de 3,0 a 3,25 (escala de 1 a 5), pois um ECC inferior a 3,0 é associado à reduzida produtividade e desempenho reprodutivo, ao passo que um ECC igual ou superior a 3,5 é associada à redução do consumo de matéria seca (CMS) no 
início do período lactacional, bem como da produção leiteira e aumento no risco de incidência de doenças metabólicas (Roche et al., 2009).

O emprego de formulações de drench em vacas periparturiente tem sido relatado e fundamentado em estudos recentes. Oetzel et al. (2012) discorreu que o emprego de Cloreto de cálcio na formulação de drench objetiva a mantença das concentrações séricas do cálcio dentro da normalidade. O cloreto de potássio também contido nas formulações auxilia na reposição de eletrólitos excretados durante o parto, fornece o principal cátion do fluido intracelular e auxilia na manutenção do equilíbrio acidobásico, retenção de água e absorção de magnésio $\left(\mathrm{Mg}^{2+}\right)$. O sulfato de magnésio, por sua vez é agente tamponante que auxilia na prevenção da hipocalcemia via fornecimento adicional de magnésio (Ammermann \& Goodrich, 1983).

\section{Considerações finais}

A hipocalcemia é uma doença metabólica que afeta a grande maioria das vacas periparturientes no Brasil e no mundo. Os maiores prejuízos se dão quando na forma subclínica da enfermidade, uma vez que não há demonstração de sinais clínicos e exige a realização de exames laboratoriais para o seu diagnóstico. Além disso a doença pode vir acometer até 50\% das vacas leiteiras dentro de um rebanho.

A prevenção, conquanto, se mostra mais atrativa, devendo ser realizado um correto manejo nutricional das vacas no período seco e período de transição. Ademais, o monitoramento do ECC é louvável para dirimir riscos de aos animais chegarem ao momento do parto acima ou abaixo do peso, aumentando, assim, o risco de doenças metabólicas decorrentes.

\section{Referências}

Albani, K., \& Silva, A. (2017). Dieta com restrição de cálcio ou aniônica em vacas leiteiras no pré-parto. Arquivos de Ciências Veterinárias e Zoologia Da UNIPAR, 20(2). https://doi.org/10.25110/arqvet.v20i2.5573

Ammerman C. B. \& Goodrich R. D. (1983). Advances in mineral nutrition in rumiants. Journal Animal Science, 57, 519-533.

Berchielli, T. T., Pires, A. V, Oliveira, S. G., \& FUNEP. (2011). Nutrição de Ruminantes (Issue 2th ed.). FUNEP.

Bernardi, A. C. C.; Machado, P. O. A., Silva, C. A. (2002). Fertilidade do solo e demanda por nutrientes no Brasil. In: Manzatto, C. M., Freitas Júnior, E. \& Peres, J. R. R. Uso agrícola dos solos brasileiros. Rio de Janeiro: Embrapa Solos. 61-77.

Caple I. W. (1991). Metabolic Disorders. In: Howard J.L. Current Veterinary Therapy: Food Animal Practice. 2.ed. Philadelphia: W.B. Saunders, 311-317.

Chapinal, N., Carson, M., Duffield, T. F., Capel, M., Godden, S., Overton, M., Santos, J. E. P., \& LeBlanc, S. J. (2011). The association of serum metabolites with clinical disease during the transition period. Journal of Dairy Science, 94(10), 4897-4903. https://doi.org/10.3168/jds.2010-4075

Corbellini, C. N. (1998). Etiopatogenia e controle da hipocalcemia e hipomagnesemia em vacas leiteiras. Seminário Internacional Sobre Deficiências Minerais Em Ruminantes, 28.

Curtis, C. R., Erb, H. N., Sniffen, C. J., Smith, R. D., Powers, P. A., Smith, M. C., White, M. E., Hillman, R. B., \& Pearson, E. J. (1983). Association of parturient hypocalcemia with eight periparturient disorders in Holstein cows. Journal of the American Veterinary Medical Association, 183(5), 559561. http://europepmc.org/abstract/MED/6618988

Dukes, H. Hugh. (1996). Dukes`Fisiologia dos Animais Domésticos. 11.ed. 421 Rio de Janeiro: Guanabara Koogan. P.

Eddy, R. G. (1999). Major metabolic disorders. In: Andrews A.H., Boyd B.H., Eddy R.G. Bovine Medicine. Malden: Blackwell Science, 577-583.

Farnia, S. A., Rasooli, A., Nouri, M., Shahryaric, A., Bakhtiaryd, M. K., Constable, P. D. (2018). Effect of postparturient oral calcium administration on serum total calcium concentration in Holstein cows fed diets of different dietary cation-anion difference in late gestation. Research in Veterinary Science. 117, 118-124. https://doi.org/10.1016/j.rvsc.2017.11.017 
Frigotto, T. A., Navarro, R. B., Schmidt, P., Barros Filho, I. R., Ollhoff, R. D., \& Almeida, R. (2010). Implicações clínicas e produtivas do $\mathrm{pH}$ urinário de vacas leiteiras de alta produção no período préparto. Archives of Veterinary Science, 15(4). https://doi.org/10.5380/avs.v15i4.16443

Goff, J. P. (2014). Calcium and magnesium disorders. Veterinary Clinics of North America - Food Animal Practice. 30, 359-381. https://doi.org/10.1016/j.cvfa.2014.04.003

Goff, J.P. (2008). The Monitoring, Prevention, And Treatment Of Milk Fever And Subclinical Hypocalcemia In Dairy Cows. The Veterinary Journal, 176(1), 50-57. Https://Doi.Org/10.1016/J.Tvj1.2007.12.020

Goff, J.P. (2004). Macromineral disorders of the transition cow. Veterinary Clinics of North America Food Animal Practice. 20, 471-494. https://doi.org/10.1016/j.cvfa.2004.06.003

Goff, J. P. (1999). Treatment of calcium, phosphorus, and magnesium balance disorders. Veterinary Clinics of North America: Food Animal Practice. 15, 619-639. https://doi.org/10.1016/s07490720(15)30167-5

González, F., Barcellos, J., Patiño, H., \& Ribeiro, L. (2000). Perfil metabólico em ruminantes: seu uso em nutrição e doenças nutricionais. UFGRS, Porto Alegre, Brasil., 89.

González, F. H. D. \& Silva, S. C. (2017). Introdução à Bioquímica Clínica Veterinária. $3^{a}$ Ed. Porto Alegre: Editora UFRGS.

Hayirli, A., Grummer, R. R., Nordheim, E. V., \& Crump, P. M. (2002). Animal and Dietary Factors Affecting Feed Intake During the Prefresh Transition Period in Holsteins. Journal of Dairy Science, 85(12), 3430-3443. https://doi.org/10.3168/jds.S0022-0302(02)74431-7

Huzzey, J. M., Veira, D. M., Weary, D. M., \& von Keyserlingk, M. A. G. (2007). Prepartum Behavior and Dry Matter Intake Identify Dairy Cows at Risk for Metritis. Journal of Dairy Science, 90(7), 3220-3233. https://doi.org/http://dx.doi.org/10.3168/jds.2006-807

IBGE. (2019). Anuário leite. Instituto Brasileiro de Geografia e Estatística, Embrapa, Juíz de Fora, Minas Gerais, Brasil.

Ingvartsen, K. L., \& Moyes, K. M. (2015). Factors contributing to immunosuppression in the dairy cow during the periparturient period. Japanese Journal of Veterinary Research, 63(Supplement 1), S15S24. https://doi.org/10.14943/jjvr.63.suppl.s15

Jawor, P.E., Huzzey, J.M., Leblanc, S.J., Von Keyserlingk, M.A.G. (2012). Associations of subclinical hypocalcemia at calving with milk yield, and feeding, drinking, and standing behaviors around parturition in Holstein cows. Journal of Dairy Science. 95, 1240-1248. https://doi.org/10.3168/jds.2011-4586

Kronfeld, D. S. (1980). Homeostatic disorders associated with lactation. In: Amstutz,H. E. Bovine medicine and surgery. 2nd ed. Santa Barbara: American Veterinary Publications, pp.565-576.

Leite, L. C., Andriguetto, J. L., Paula, M. C., \& Rocha, R. M. V. M. (2003). Diferentes balanços catiônicos-aniônicos da dieta de vacas da raça holandesa. Revista Brasileira de Zootecnia, 32(5), $1259-1265$.

Lean, I. J., Degaris, P. J., Mcneil, D. M., Block, E. (2006). Hypocalemia in Dairy Cows: Metaanalysis and Dietary Cation Anion Difference Theory Revisited. Journal of Dairy Science. 89, 669-684. https://doi.org/10.3168/jds.s0022-0302(06)72130-0

Liesegang, A., Sassi, M. L., Risteli, J., Eicher, R., Wanner, M. \& Riond J.L. (1998). Comparison of bone resorption markers during hypocalcemia in dairy cows. Journal Dairy Science. 81: 2614-2622. https://doi.org/10.3168/jds.s0022-0302(98)75819-9

Littledike, E. T., \& Goff, J. (1987). Interactions of Calcium, Phosphorus, Magnesium and Vitamin D that Influence their Status in Domestic Meat Animals. Journal of Animal Science, 65(6), 1727-1743. https://doi.org/10.2527/jas1987.6561727x

Martín-Tereso, J., \& Martens, H. (2014). Calcium and Magnesium Physiology and Nutrition in Relation to the Prevention of Milk Fever and Tetany (Dietary Management of Macrominerals in Preventing Disease). Veterinary Clinics of North America: Food Animal Practice, 30(3), 643-670. https://doi.org/10.1016/j.cvfa.2014.07.007

Martinez, N., Risco, C. A., Lima, F. S., Bisinotto, R. S., Greco, L. F., Ribeiro, E. S., Maunsell, F., Galvão, K., \& Santos, J. E. P. (2012). Evaluation of peripartal calcium status, energetic profile, and 
neutrophil function in dairy cows at low or high risk of developing uterine disease. Journal of Dairy Science, 95(12), 7158-7172. https://doi.org/10.3168/jds.2012-5812

Martinez, N., Sinedino, L. D. P., Bisinotto, R. S., Ribeiro, E. S., Gomes, G. C., Lima, F. S., Greco, L. F., Risco, C. A., Galvão, K. N., Taylor-Rodriguez, D., Driver, J. P., Thatcher, W. W., \& Santos, J. E. P. (2014). Effect of induced subclinical hypocalcemia on physiological responses and neutrophil function in dairy cows. Journal of Dairy Science, 97(2), 874-887. https://doi.org/10.3168/jds.20137408

Moreira, T. F., Zambrano, J. U., Paula, V. M., Casagrande, F. P., Facury Filho, E. J., Molina, L. R., Leme, F. O. P., \& Carvalho, A. Ú. (2015). Perfil mineral de vacas mestiças Girolanda no período de transição em sistema semi-intensivo em duas estações do ano.

Mulligan, F. J., O'grady, L. \& Doherty, M. L. (2008). Milk fever and subclinical hypocalcemia: their role in transition cow health and prevention strategies. British Cattle Veterinary Association, 16, 188193.

Oetzel G.R. 2013. Oral calcium supplementation in peripartum dairy cows. Veterinary Clinics of North America: Food Animal Practiceet, 29(2):447-455. https://doi.org/10.1016/j.cvfa.2013.03.006

Oetzel, G. R. (2012). An update on hypocalcemia on dairy farms. Proceedings of the Four-State Dairy Nutrition and Management Conference, 80-85.

Ogilvie T.H. (2000). Distúrbios metabólicos. In: Ogilvie, T. H. Medicina interna de grandes animais. Porto Alegre: Artes Médicas, 225-227.

Oliveira, S. G., Berchielli, T. T. \& Pires, A. V. (2011). Nutrição de Ruminantes. 2. ed. Funep, Jaboticabal, Brasil.

Ortolani E.L. (1995). Aspectos clínicos, epidemiológicos e terapêuticos da 491 hipocalcemia de vacas leiteiras. Arquivo Brasileiro de Medicina Veterinária e Zootecnia, Belo Horizonte, 47, 799-808.

Overton, T. R., \& Waldron, M. R. (2004). Nutritional Management of Transition Dairy Cows: Strategies to Optimize Metabolic Health. Journal of Dairy Science, 87, E105-E119. https://doi.org/10.3168/jds.S0022-0302(04)70066-1

Pires, A. V, Ribeiro, C. V. M., Mendes, C. Q., \& FUNEP. (2011). Aspectos nutricionais relacionados a reprodução. In T. T. Berchielli, A. V Pires, \& S. G. Oliveira (Eds.), Nutrição de Ruminantes (Vol. 1, Issue 2th ed., pp. 537-564). FUNEP.

Radostits, O. M., Gay, C. C., Blood, D. C. \& Hinchcliff, K.W. (2002). Clínica Veterinária: um tratado de doenças dos bovinos, ovinos, suinos, caprinos e equinos, $9^{\mathrm{a}}$ ed. Rio de Janeiro: Guanabara Koogan.

Reinhardt, T. A., Lippolis, J. D., Mccluskey, B. J., Goff, J. P. \& Horst R. L. (2011). The Prevalence of subclinical hypocalcemia in dairy herds. Veterinary Journal. 188, 122- 124. https://doi.org/10.1016/j.tvjl.2010.03.025

Riet-Correa, F. Outras doenças. Riet-Correa, F. et al. (2001). In: Doenças de Ruminantes e Equinos. 2 ed. São Paulo: Livraria Varela, v. 2, cap. 7, 523-525.

Riet-Correa, F., Méndez, F., Carmen Schild, M., Riet-Correa, A. L. F., Schild, A. L., \& Carmen, M. M. (2007). Doenças de ruminantes e eqüinos. Varela.

Roche, J. R., Friggens, N. C., Kay, J. K., Fisher, M. W., Stafford, K. J., \& Berry, D. P. (2009). Invited review: Body condition score and its association with dairy cow productivity, health, and welfare. Journal of Dairy Science, 92(12), 5769-5801. https://doi.org/10.3168/jds.2009-2431

Santos, J. E. P. (2011). Distúrbios metabólicos. In T. T. Berchielli, A. V Pires, \& S. G. Oliveira (Eds.), Nutrição de Ruminantes- FUNEP (Vol. 1, Issue 2th ed., pp. 439-520). FUNEP.

Schafhäuser Jr, J. (2006). Balanço de cátions e ânions em dietas para vacas leiteiras no período de transição. Revista Da FZVA, 13(1).

Silveira, P. A., Fensterseifer, S., Pereira, R. A., Schneider, A., Bianchi, I., \& Corrêa, M. N. (2009). Impacto econômico das doenças do periparto de vacas leiteiras. Núcleo de Pesquisa, Ensino $e$ Extensão Em Pecuária (NUPEEC).

Smith, B. P. (2006). Tratado de Medicina Interna de Grandes Animais (3. ed. Man). 
Van Cleef, E., Patiño, R., Neiva Jr, A., Serafim, R., Rego, A. \& Gonçalves, J. (2009). Distúrbios metabólicos por manejo alimentar inadequado em ruminantes: novos conceitos, Revista Colombiana Ciência Animal. 1, 319-341. https://doi.org/10.24188/recia.v1.n2.2009.376

Vilela, D., Leite, J. L. B., \& Resende, J. C. (2002). Políticas para o leite no Brasil: passado, presente e futuro. Simpósio Sobre Sustentabilidade da Pecuária Leiteira na Região Sul do Brasil, 1, 1-26.

Wilkens M.R., Praechter C., Breves G. \& Schröder B. (2016). Stimulating effects of a diet negative in dietary cation-anion difference on calcium absorption from the rumen in sheep. Journal of Animal Physiology and Animal Nutrition, 100(1):156-166.

Wu, W. X., Liu, J. X., Xu, G. Z., \& Ye, J. A. (2008). Calcium homeostasis, acid-base balance, and health status in periparturient Holstein cows fed diets with low cation-anion difference. Livestock Science, 117(1), 7-14. https://doi.org/10.1016/j.livsci.2007.11.005

\section{Histórico do artigo}

Recebido: 1 de outubro de 2020

Aprovado: 23 de outubro de 2020.

Licenciamento: Este artigo é publicado na modalidade Acesso Aberto sob a licença Creative Commons Disponível online: 27 de dezembro de 2020. 